\title{
Change in N-terminal pro-B-type natriuretic peptide at 1 year predicts mortality in wild-type transthyretin amyloid cardiomyopathy
}

\author{
Steven Law (1), ${ }^{1}$ Aviva Petrie, ${ }^{2}$ Liza Chacko, ${ }^{1}$ Oliver C Cohen, ${ }^{1}$ Sriram Ravichandran, ${ }^{1}$ \\ Janet A Gilbertson, ${ }^{1}$ Dorota Rowczenio, ${ }^{1}$ Ashutosh D Wechalekar, ${ }^{1}$ \\ Ana Martinez-Naharro, ${ }^{1}$ Helen J Lachmann, ${ }^{1}$ Carol J Whelan, ${ }^{1}$ David F Hutt (ㄷ, , \\ Philip N Hawkins, ${ }^{1}$ Marianna Fontana, ${ }^{1}$ Julian D Gillmore ${ }^{1}$
}

${ }^{1}$ National Amyloidosis Centre, Division of Medicine, University College London, London, UK ${ }^{2}$ Eastman Dental Institute, University College London, London, UK

\section{Correspondence to}

Dr Julian D Gillmore, Division of Medicine, University College London, London WC1E 6BT, UK; j.gillmore@ucl.ac.uk

Received 24 January 2021 Revised 15 April 2021 Accepted 20 April 2021

\section{Check for updates}

(c) Author(s) (or their employer(s)) 2021. Re-use permitted under CC BY-NC. No commercial re-use. See rights and permissions. Published by BMJ.

To cite: Law S, Petrie A,

Chacko L, et al. Heart

Epub ahead of print:

[please include Day Month

Year]. doi:10.1136/

heartinl-2021-319063

\section{ABSTRACT}

Objectives Wild-type transthyretin amyloid cardiomyopathy (wtATTR-CM) is a progressive and fatal condition. Although prognosis can be determined at the time of diagnosis according to National Amyloidosis Centre (NAC) transthyretin amyloidosis (ATTR) stage, the clinical course varies substantially between individuals. There are currently no established measures of rate of disease progression. Through systematic analysis of functional, biochemical and echocardiographic diseaserelated variables we aimed to identify prognostic markers of disease progression in wtATTR-CM.

Methods This is a retrospective observational study of 432 patients with wtATTR-CM diagnosed at the UK $N A C$, none of whom received disease-modifying therapy. The association between mortality from the 12-month timepoint and change from diagnosis to 12 months in a variety of disease-related variables was explored using Cox regression.

Results Change in N-terminal pro-B-type natriuretic peptide concentration ( $\triangle$ NT-proBNP) at 12 months from diagnosis was the strongest predictor of ongoing mortality and was independent of both change in other disease-related variables (HR 1.04 per $500 \mathrm{ng} / \mathrm{L}$ increase ( $95 \% \mathrm{Cl} 1.01$ to 1.07$) ; p=0.003$ ) and a range of known prognostic variables at the time of diagnosis (HR 1.07 per $500 \mathrm{ng} / \mathrm{L}$ increase $(95 \% \mathrm{Cl} 1.02$ to 1.13$) ; p=0.007)$. An increase in NT-proBNP of $>500 \mathrm{ng} / \mathrm{L},>1000 \mathrm{ng} / \mathrm{L}$ and $>2000 \mathrm{ng} / \mathrm{L}$ during the first year of follow-up occurred in $45 \%, 35 \%$ and $16 \%$ of patients, respectively.

Conclusion Change in NT-proBNP concentration during the first year of follow-up is a powerful independent predictor of mortality in wtATTR-CM.

\section{INTRODUCTION}

Wild-type transthyretin amyloid cardiomyopathy (wtATTR-CM) is an increasingly recognised cause of heart failure. The exact prevalence of wtATTR-CM remains unknown, but high-grade cardiac uptake on ${ }^{99 \mathrm{~m}}$ Technetium labelled 3,3-diphosphon o-1,2-propanodicarboxylic acid (Tc-DPD) scintigraphy was reported in $3.9 \%$ of men over 75 years of age in a recent Spanish study. ${ }^{1}$ Advances in imaging techniques $^{2-4}$ and development of validated nonbiopsy diagnostic criteria for ATTR-CM ${ }^{5}$ have led to a recent exponential rise in diagnoses of wtATTR-CM throughout the world.

Diagnosis of wtATTR-CM is often delayed and may occur at any time during the disease natural history, ${ }^{78}$ which is of inexorable progression and death within 10 years of diagnosis. ${ }^{7}$ Prognosis can be estimated at the time of diagnosis by stratifying patients by the National Amyloidosis Centre (NAC) transthyretin amyloidosis (ATTR) stage according to the $\mathrm{N}$-terminal pro-B-type natriuretic peptide (NT-proBNP) concentration and estimated Modification of Diet in Renal Disease (MDRD) glomerular filtration rate (eGFR). ${ }^{9}{ }^{10}$ However, there is substantial interpatient variability in the rate of disease progression of wtATTR-CM such that there is an urgent need for a widely applicable marker of disease progression in this population.

We sought to evaluate the change between diagnosis and 12 months of follow-up in a range of disease-related variables to identify the best marker of disease progression and prognosis in wtATTR-CM.

\section{METHODS}

\section{Study design}

This is a retrospective observational cohort study of 432 patients with symptomatic wtATTR-CM diagnosed at the UK NAC between June 2006 and October 2018. Patients with hereditary ATTR amyloidosis and those receiving any form of diseasemodifying therapy were excluded; no patients underwent heart transplantation. Diagnosis was established at NAC in all patients by Tc-DPD scintigraphy coupled with biochemical tests for a clonal dyscrasia on the basis of validated criteria, ${ }^{511}$ or by histology including immunohistochemistry and proteomic analysis of amyloid, undertaken at NAC. The diagnostic clinical evaluation at NAC as well as routine clinical follow-up performed $12 \pm 3$ months later also included a full biochemical profile (renal, liver, bone, cardiac biomarkers), echocardiography, and functional assessment including NYHA class and 6 min walk test (6MWT).

All patients provided informed consent for anonymous publication of their data. Patients or the public were not involved in the design, or conduct, or reporting or dissemination plans of our research. 


\section{Biomarker analysis}

NT-proBNP was measured with an electrochemiluminescence sandwich immunoassay on the Elecsys 2010 system (Roche Diagnostics). eGFR was calculated by standard MDRD study equation including correction for race.

\section{Statistical methods}

Date of diagnosis (baseline) was defined as date of first review at NAC, and patients were censored at death or last clinical contact. Mortality data were obtained from the National Health Service central care records. Censor date was 18 October 2019.

Change in a range of disease-related variables (labelled as $\Delta$ variable throughout the manuscript) between baseline and 12 months was calculated, and the association between each $\Delta$ variable and mortality from the 12-month timepoint was explored by univariable Cox regression analyses and subsequently by a multivariable Cox regression analysis in which the best performing biochemical (NT-proBNP), echocardiographic (interventricular septal thickness at end diastole, IVSd) and functional (New York Heart Association (NYHA) class) variables significant in the univariable analyses were included. The 12-month timepoint was selected to identify an early marker of disease progression which would be reached by the majority of patients diagnosed with ATTR-CM; it also matches the timepoint of the primary endpoint measure being used in part $\mathrm{A}$ of the ongoing phase III clinical trial ATTRibute-CM. Time-dependent receiver operator characteristic (ROC) curve analyses were performed using both absolute $\Delta$ NT-proBNP concentration from baseline and percentage $\Delta$ NT-proBNP from baseline to identify the optimal measure of $\Delta$ NT-proBNP. The area under the curve was higher for absolute $\Delta$ NT-proBNP than for percentage $\Delta$ NT-proBNP (0.63 vs 0.60 ); therefore, absolute $\Delta$ NT-proBNP was used for all subsequent analyses. The relationship between $\Delta$ NT-proBNP and mortality from the 12-month timepoint was further explored by multivariable Cox regression analyses including a range of previously reported prognostic factors assessed at diagnosis. ${ }^{12}$ Landmark Kaplan-Meier survival curves illustrate survival from the 12-month timepoint stratified by different $\Delta$ NT-proBNP values.

Data are presented as median (IQR) or number (percentage) unless otherwise stated. A $p$ value of $<0.05$ was deemed significant. Summary statistics were obtained using SPSS V.25 and all other analyses were performed using Stata V.16.

\section{RESULTS}

\section{Patient characteristics}

The baseline characteristics of all 432 patients are shown in table 1 . The median age at diagnosis of wtATTR-CM was 77 years and $95 \%$ were male. The majority of patients $(69 \%)$ had NYHA class II heart failure at diagnosis and the median 6MWT distance was $358 \mathrm{~m}$. The median (IQR) time from diagnosis to $12 \pm 3$ month follow-up timepoint was 12 (11-13) months, and the median (IQR) follow-up from the 12-month timepoint was 19 (10-31) months. At censor, 146 patients had died and 286 were alive.

\section{Survival impact of change in disease-related variables from baseline to 12 months}

Univariable Cox regression analyses identified an association between mortality from the 12-month timepoint and $\Delta$ NT-proBNP $(\mathrm{p}=0.001)$ and $\Delta$ NYHA class $(\mathrm{p}=0.005)($ table 2$)$. Multivariable analysis showed both $\triangle$ NT-proBNP (HR 1.04 (95\% CI 1.01 to 1.07 ) per $500 \mathrm{ng} / \mathrm{L}$ increase; $\mathrm{p}=0.003$ ) and increasing NYHA class (HR 1.65 (95\% CI 1.11 to 2.47);

\begin{tabular}{|c|c|c|}
\hline & n & wtATTR-CM $(\mathrm{N}=432)$ \\
\hline Age at diagnosis (years) & 432 & $77(73-82)$ \\
\hline Male gender & 432 & $409(95)$ \\
\hline Caucasian ancestry & 432 & $402(93)$ \\
\hline NAC ATTR stage I & 432 & $210(49)$ \\
\hline NAC ATTR stage II & 432 & $161(37)$ \\
\hline NAC ATTR stage III & 432 & $61(14)$ \\
\hline NT-proBNP (ng/L) & 432 & $2760(1568-4904)$ \\
\hline eGFR (MDRD, mL/min) & 432 & $60(49-74)$ \\
\hline CKD stage $\leq 1$ & & $24(6)$ \\
\hline CKD stage II & & $196(45)$ \\
\hline CKD stage IIla & & $130(30)$ \\
\hline CKD stage IIllb & & $66(15)$ \\
\hline CKD stage IV & & $16(4)$ \\
\hline CKD stage $V$ & & $0(0)$ \\
\hline Troponin T (ng/L) & 389 & $58(40-81)$ \\
\hline Serum albumin (g/L) & 432 & $44(42-46)$ \\
\hline NYHA heart failure class & 432 & \\
\hline I & & $53(12)$ \\
\hline II & & $298(69)$ \\
\hline III & & $77(18)$ \\
\hline IV & & $4(1)$ \\
\hline Comorbidities & 432 & \\
\hline Hypertension & & $147(34)$ \\
\hline Atrial fibrillation & & $226(52)$ \\
\hline Diabetes mellitus & & $54(13)$ \\
\hline Pacemaker & & $66(15)$ \\
\hline Systolic blood pressure $(\mathrm{mm} \mathrm{Hg})$ & 432 & $122(111-135)$ \\
\hline Diastolic blood pressure (mm Hg) & 432 & $72(67-79)$ \\
\hline Body mass index $\left(\mathrm{kg} / \mathrm{m}^{2}\right)$ & 432 & $26(24-29)$ \\
\hline IVSd (mm) & 427 & $17(16-18)$ \\
\hline LVPWd (mm) & 427 & $16(15-18)$ \\
\hline Left ventricular ejection fraction (\%) & 423 & $48(42-56)$ \\
\hline 6MWT (m) & 313 & $358(230-449)$ \\
\hline Perugini grade on DPD scan & 380 & \\
\hline Grade 2 & & $349(92)$ \\
\hline Grade 3 & & $31(8)$ \\
\hline
\end{tabular}

Results displayed as number (percentage) for categorical variables and median (IQR) for numerical variables.

CKD, chronic kidney disease; DPD, 3,3-diphosphono-1,2-propanodicarboxylic acid; eGFR, estimated glomerular filtration rate; IVSd, interventricular septal thickness at end diastole; LVPWd, left ventricular posterior wall thickness at end diastole; MDRD, Modification of Diet in Renal Disease; 6MWT, 6 min walk test; NAC ATTR stage, National Amyloidosis Centre transthyretin amyloidosis stage; NT-proBNP, N-terminal pro-B-type natriuretic peptide; NYHA, New York Heart Association; wtATTR-CM, wild-type transthyretin amyloid cardiomyopathy.

$\mathrm{p}=0.014)$ to be predictive of mortality from the 12 -month timepoint, independent of change in other disease-related variables (table 2).

Multivariable Cox regression analysis incorporating $\Delta$ NT-proBNP along with a range of baseline variables which are known to be prognostic in wtATTR-CM at the time of diagnosis also showed $\triangle$ NT-proBNP (HR 1.07 (95\% CI 1.02 to 1.13) per $500 \mathrm{ng} / \mathrm{L}$ increase; $\mathrm{p}=0.007$; table 3 ) to be an independent predictor of mortality along with age and NT-proBNP concentration at diagnosis. When diagnostic eGFR and NT-proBNP as individual variables were exchanged for diagnostic NAC ATTR stage (which is calculated from the same two variables) and this multivariable analysis was repeated, $\Delta$ NT-proBNP continued to 
Table 2 Association between mortality and change $(\Delta)$ from baseline to 12 months in disease-related variables by Cox regression analyses

\begin{tabular}{|c|c|c|c|c|c|c|c|}
\hline \multirow[b]{2}{*}{ Variable } & \multirow[b]{2}{*}{ Median change (IQR) } & \multicolumn{3}{|c|}{ Univariable analysis } & \multicolumn{3}{|c|}{ Multivariable analysis } \\
\hline & & HR & $95 \% \mathrm{Cl}$ & $P$ value & HR & $95 \% \mathrm{Cl}$ & $P$ value \\
\hline$\triangle \mathrm{NT}-$ proBNP (ng/L) & $375(-258$ to 1350$)$ & $1.05^{*}$ & 1.02 to 1.07 & 0.001 & $1.04^{*}$ & 1.01 to 1.07 & 0.003 \\
\hline$\triangle \mathrm{eGFR}(\mathrm{mL} / \mathrm{min})$ & $-5(-12$ to 1$)$ & 1.00 & 0.98 to 1.01 & 0.732 & & & \\
\hline$\triangle$ Albumin (g/L) & $-1(-3$ to 1$)$ & 1.01 & 0.95 to 1.06 & 0.840 & & & \\
\hline$\Delta$ Troponin $\mathrm{T}(\mathrm{ng} / \mathrm{L})$ & 12 (4 to 25$)$ & 1.01 & 1.00 to 1.02 & 0.055 & & & \\
\hline$\Delta \mathrm{IVSd}(\mathrm{mm})$ & 0 (0 to 1$)$ & 1.15 & 0.99 to 1.34 & 0.075 & 1.03 & 0.85 to 1.25 & 0.741 \\
\hline$\triangle$ LVPWd (mm) & $0(0$ to 1$)$ & 1.12 & 0.98 to 1.27 & 0.099 & & & \\
\hline$\triangle \operatorname{LVEF}(\%)$ & $-1(-6$ to 4$)$ & 1.00 & 0.98 to 1.02 & 0.886 & & & \\
\hline Increasing NYHA class & $0(0$ to 1$)$ & 1.72 & 1.18 to 2.52 & 0.005 & 1.65 & 1.11 to 2.47 & 0.014 \\
\hline$\triangle$ Systolic blood pressure $(\mathrm{mm} \mathrm{Hg})$ & $-1(-11$ to 9$)$ & 1.00 & 0.99 to 1.01 & 0.741 & & & \\
\hline$\Delta$ Diastolic blood pressure $(\mathrm{mm} \mathrm{Hg})$ & $1(-7$ to 7$)$ & 1.00 & 0.98 to 1.01 & 0.556 & & & \\
\hline$\triangle$ NAC ATTR stage & $0(0$ to 0$)$ & 1.39 & 0.94 to 2.06 & 0.096 & & & \\
\hline$\Delta 6 \mathrm{MWT}(\mathrm{m})$ & $-11(-70$ to 16$)$ & $0.85 t$ & 0.70 to 1.02 & 0.080 & & & \\
\hline
\end{tabular}

Increasing NYHA class is displayed as a binary measure.

P-values meeting statistical significance $(\mathrm{p}<0.05)$ are highlighted in bold.

${ }^{*} \mathrm{HR}$ for NT-proBNP is per $500 \mathrm{ng} / \mathrm{L}$ increase.

THR for 6MWT distance is per $100 \mathrm{~m}$ increase; the remainder of HRs are per unit increase.

eGFR, estimated glomerular filtration rate; IVSd, interventricular septal thickness at end diastole; LVEF, left ventricular ejection fraction; LVPWd, left ventricular posterior wall

thickness at end diastole; 6MWT, 6 min walk test; NAC ATTR stage, National Amyloidosis Centre transthyretin amyloidosis stage; NT-proBNP, N-terminal pro-B-type natriuretic

peptide; NYHA, New York Heart Association.

predict mortality (HR 1.07 (95\% CI 1.01 to 1.14$)$ per $500 \mathrm{ng} / \mathrm{L}$ increase; $\mathrm{p}=0.017)$.

Time-dependent ROC curve analyses identified absolute $\Delta$ NT-proBNP concentration from baseline to be a better predictor of mortality than percentage $\Delta$ NT-proBNP. The median $\Delta$ NT-proBNP was not significantly different between different diagnostic NAC ATTR stages $(\mathrm{p}=0.19$ by Kruskal-Wallis test), and there was no evidence of an association between $\Delta$

Table 3 Multivariable analysis including $\triangle$ NT-proBNP at 12 months and a range of variables and baseline patient characteristics known to affect prognosis

\begin{tabular}{|c|c|c|c|c|}
\hline & & HR & $95 \% \mathrm{Cl}$ & $P$ value \\
\hline \multicolumn{2}{|c|}{$\triangle$ NT-proBNP at 12 months* } & 1.07 & 1.02 to 1.13 & 0.007 \\
\hline \multicolumn{2}{|c|}{ Diagnostic NT-proBNP (ng/L)* } & 1.07 & 1.02 to 1.13 & 0.006 \\
\hline \multicolumn{2}{|c|}{ Diagnostic troponin $\mathrm{T}$ (ng/L) } & 1.01 & 1.00 to 1.02 & 0.147 \\
\hline \multicolumn{2}{|c|}{ Diagnostic eGFR $\left(\mathrm{mL} / \mathrm{min} / 1.73 \mathrm{~m}^{2}\right)$} & 1.01 & 0.99 to 1.03 & 0.377 \\
\hline \multicolumn{2}{|c|}{ Age at diagnosis } & 1.08 & 1.02 to 1.13 & 0.004 \\
\hline \multirow[t]{3}{*}{ NYHA class at diagnosis } & 1 & 1 & & \\
\hline & $\|$ & 0.74 & 0.33 to 1.65 & 0.461 \\
\hline & $\geq I I I$ & 0.39 & 0.14 to 1.15 & 0.089 \\
\hline \multicolumn{2}{|l|}{ IVSd at diagnosis } & 0.93 & 0.81 to 1.07 & 0.321 \\
\hline \multicolumn{2}{|l|}{ Body mass index $\left(\mathrm{kg} / \mathrm{m}^{2}\right)$} & 0.95 & 0.88 to 1.03 & 0.244 \\
\hline \multicolumn{2}{|c|}{6 min walk test distance at diagnosis $(\mathrm{m}) \dagger$} & 0.91 & 0.72 to 1.16 & 0.457 \\
\hline \multicolumn{2}{|c|}{ Atrial fibrillation } & 0.90 & 0.50 to 1.63 & 0.738 \\
\hline \multicolumn{2}{|l|}{ Hypertension } & 1.01 & 0.54 to 1.89 & 0.986 \\
\hline \multicolumn{2}{|l|}{ Diabetes } & 2.19 & 0.99 to 4.86 & 0.054 \\
\hline \multicolumn{2}{|c|}{ Permanent pacemaker in situ } & 0.73 & 0.31 to 1.68 & 0.454 \\
\hline \multicolumn{2}{|l|}{ Aortic stenosisł } & 1.47 & 0.53 to 4.11 & 0.459 \\
\hline
\end{tabular}

$\triangle$ NT-proBNP was calculated at the 12-month timepoint; all other variables were assessed at diagnosis.

P-values reaching statistical significance $(p<0.05)$ are highlighted in bold

${ }^{*} \mathrm{HR}$ for NT-proBNP is per $500 \mathrm{ng} / \mathrm{L}$ increase.

tHR for 6MWT distance is per $100 \mathrm{~m}$ increase.

$\ddagger \geq$ Moderate aortic stenosis at diagnosis; the remainder of HRs are per unit increase. eGFR, estimated glomerular filtration rate; IVSd, interventricular septal thickness at end diastole; 6MWT, 6 min walk test; NT-proBNP, N-terminal pro-B-type natriuretic peptide; NYHA, New York Heart Association.
NT-proBNP and the follow-up interval from diagnosis, which ranged from 9 to 15 months $(\mathrm{p}=0.13)$. The proportion of patients with $\Delta$ NT-proBNP $>500 \mathrm{ng} / \mathrm{L},>1000 \mathrm{ng} / \mathrm{L}$ and $>2000 \mathrm{ng} / \mathrm{L}$ at 12 months was $45 \%, 35 \%$ and $16 \%$, respectively. Landmark Kaplan-Meier survival curves stratified for $\Delta$ NT-proBNP cutoffs of $>500 \mathrm{ng} / \mathrm{L}$ or $\leq 500 \mathrm{ng} / \mathrm{L},>1000 \mathrm{ng} / \mathrm{L}$ or $\leq 1000 \mathrm{ng} / \mathrm{L}$, and $>2000 \mathrm{ng} / \mathrm{L}$ or $\leq 2000 \mathrm{ng} / \mathrm{L}$ are shown in figure 1 . Among the 432 patients, 150 (35\%) with $\Delta$ NT-proBNP $>1000 \mathrm{ng} / \mathrm{L}$ had a median survival of 29 (95\% CI 25 to 33 ) months compared with 46 (95\% CI 38 to 54) months in 282 of 432 (65\%) patients with $\Delta$ NT-proBNP $\leq 1000 \mathrm{ng} / \mathrm{L}(\mathrm{p}<0.001)$. Both $\Delta$ NT-proBNP $>500 \mathrm{ng} / \mathrm{L}$ (HR 1.65 (95\% CI 1.18 to 2.31 ); $\mathrm{p}=0.003$ ) and $>2000 \mathrm{ng} / \mathrm{L}$ (HR 2.87 (95\% CI 1.93 to 4.27 ); $\mathrm{p}<0.001$ ) also predicted ongoing mortality.

\section{DISCUSSION}

This study establishes $\Delta$ NT-proBNP at 12 months from diagnosis as a powerful independent predictor of ongoing mortality in patients with wtATTR-CM. Importantly, the prognostic relevance of $\Delta$ NT-proBNP was independent of a range of biochemical, functional and echocardiographic parameters at diagnosis, including NAC ATTR stage, troponin T, age, NYHA class, 6MWT distance, presence of atrial fibrillation and IVSd thickness.

Limitations of our study include its retrospective design, exclusion of patients who die within the first year of follow-up (approximately 5\% of patients in the UK), exclusion of more detailed echocardiographic variables and absence of cardiac magnetic resonance data. ${ }^{13-16}$ However, the authors maintain that a biomarker-based prognostic system has great attraction due to its simplicity, lack of operator variability, and universal availability and applicability. There may also be concerns that NT-proBNP concentration is known to be confounded by other factors in the wtATTR-CM population; however, where possible, these were accounted for in our multivariable model. Furthermore, in systemic light chain cardiac amyloidosis, a far more heterogeneous disease than wtATTR-CM, diagnostic NT-proBNP predicts mortality and both response to treatment and disease progression are defined by $\Delta$ NT-proBNP, which is 


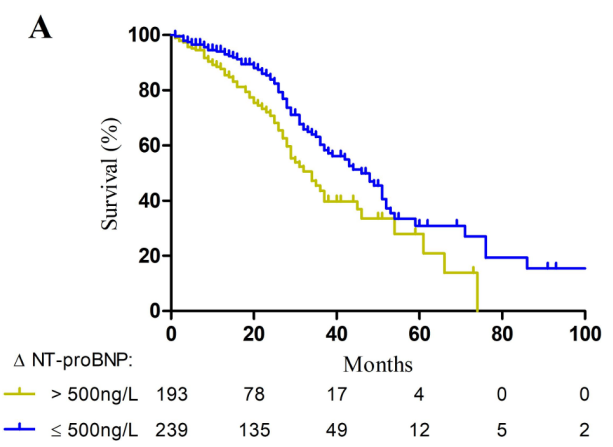

B

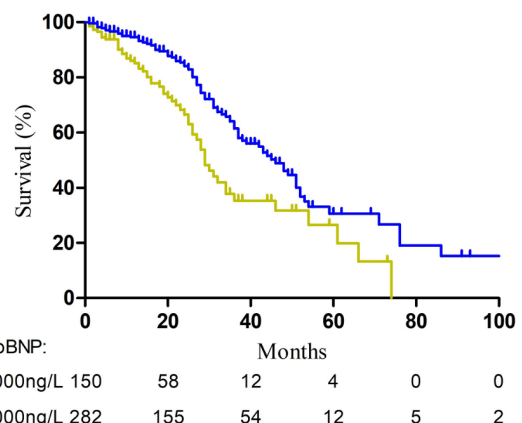

C

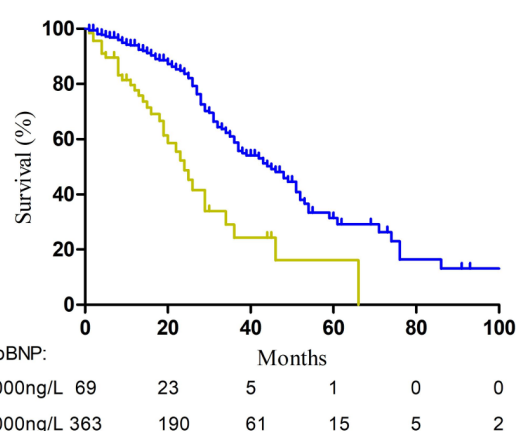

Figure 1 Landmark Kaplan-Meier survival curves stratified by $\Delta$ NT-proBNP during the first year of follow-up. Numbers at risk are shown below each curve. (A) Patient survival stratified by $\triangle$ NT-proBNP $>500 \mathrm{ng} / \mathrm{L}$ or $\leq 500 \mathrm{ng} / \mathrm{L}$ (HR 1.65 (95\% Cl 1.18 to 2.31 ); $\mathrm{p}=0.003$ ). (B) Patient survival stratified by $\triangle$ NT-proBNP $>1000 \mathrm{ng} / \mathrm{L}$ or $\leq 1000 \mathrm{ng} / \mathrm{L}$ (HR 1.92 (95\% Cl 1.37 to 2.70); $\mathrm{p}<0.001)$. (C) Patient survival stratified by $\triangle$ NT-proBNP $>2000 \mathrm{ng} / \mathrm{L}$ or $\leq 2000 \mathrm{ng} / \mathrm{L}$ (HR 2.87 (95\% Cl 1.93 to 4.27); $p<0.001)$. NT-proBNP, N-terminal pro-B-type natriuretic peptide.

strongly associated with morbidity, quality of life and mortality despite the same, and indeed additional, confounders. ${ }^{17}{ }^{18}$ Our study does not include patients on disease-modifying therapy since tafamidis is not available in the UK.

A number of disease-modifying therapies have emerged for ATTR amyloidosis, including diflunisal, ${ }^{19}$ tafamidis ${ }^{20}$ pati$\operatorname{siran}^{21} 22$ and inotersen, ${ }^{2324}$ and several of the relevant clinical trials suggest a role of $\Delta$ NT-proBNP in the assessment of response to these disease-modifying treatments. Tafamidis is associated with a reduction in mortality among patients with ATTR-CM, and the ATTR-ACT trial showed a smaller increase in NT-proBNP in the tafamidis group compared with placebo. ${ }^{20}$ Post-hoc analysis of the subpopulation with hereditary ATTR-CM accompanying neuropathy in the APOLLO study showed a highly significant reduction in NT-proBNP concentration among patients receiving patisiran compared with those on placebo. ${ }^{22}$ Of note, the trial design of ATTRibute-CM, a current global phase III clinical trial evaluating the novel transthyretin
Key messages

What is already known on this subject?

- The natural history of wild-type transthyretin amyloid cardiomyopathy (wtATTR-CM) is of inexorable progression and death within 10 years of diagnosis.

- Prognosis in the absence of disease-modifying treatment is predicted at diagnosis by the National Amyloidosis Centre transthyretin amyloidosis (ATTR) stage, although there is significant interpatient variability in the rate of disease progression.

- At present there are no established markers of disease progression widely applicable to the wtATTR-CM population.

\section{What might this study add?}

- This study establishes change in N-terminal pro-B-type natriuretic peptide in the first year after diagnosis ( $\triangle$ NTproBNP) as a powerful independent predictor of mortality in wtATTR-CM.

\section{How might this impact on clinical practice?}

- This study highlights a role of $\triangle$ NT-proBNP at 12 months in identifying patients with a more aggressive disease phenotype.

- Further study in a cohort receiving disease-modifying treatment is warranted to assess the utility of $\triangle$ NT-proBNP as a marker of treatment response and potential surrogate clinical trial endpoint.

(TTR) stabiliser acoramidis in ATTR-CM, includes a part A for which the primary endpoint is a comparison of $\Delta$ 6MWT distance from enrolment to 12 months between the treatment and placebo groups. ${ }^{25}$ Although $\Delta 6 \mathrm{MWT}$ distance undoubtedly provides a valuable functional assessment, it is noteworthy that it did not predict survival in our cohort.

On the basis of these data, we believe that evaluation of $\Delta$ NT-proBNP at 12 months in a cohort with wtATTR-CM receiving disease-modifying therapy is warranted to further assess its potential utility as a marker of treatment response and potential use as a surrogate endpoint in future clinical trials. Multiple disease-modifying therapies may soon be available for wtATTR-CM, including both gene silencer and TTR stabiliser therapies, and early markers of treatment response will be required to guide therapeutic decisions.

In summary, we establish for the first time that $\Delta$ NT-proBNP concentration in the first year after diagnosis of wtATTR-CM is a powerful independent predictor of ongoing mortality. Further study of $\Delta$ NT-proBNP in a wtATTR-CM cohort receiving disease-modifying therapy is warranted to establish its potential utility as an indicator of treatment response.

Acknowledgements We thank our many physician colleagues for referring the patients.

Contributors SL, AP, MF and JDG were responsible for conceiving the study, interpreting the results and drafting the manuscript. LC, OCC, SR, JAG, DR, ADW, AM$\mathrm{N}, \mathrm{HJL}, \mathrm{CJW}, \mathrm{DFH}$ and PNH were responsible for data collection and interpretation. MF and JDG are joint last authors of this work.

Funding The authors have not declared a specific grant for this research from any funding agency in the public, commercial or not-for-profit sectors.

Competing interests JDG is an Expert Advisory Board member for Akcea, Alnylam and Eidos. ADW and DFH report personal fees from Akcea, outside the submitted work. The remaining authors have nothing to disclose. 
Patient and public involvement Patients and/or the public were not involved in the design, or conduct, or reporting, or dissemination plans of this research.

Patient consent for publication Not required.

Ethics approval All patients were managed in accordance with the Declaration of Helsinki. The study received IRB approval from the Royal Free Hospital Ethics Committee.

Provenance and peer review Not commissioned; externally peer reviewed. Data availability statement Data are available upon reasonable request.

Open access This is an open access article distributed in accordance with the Creative Commons Attribution Non Commercial (CC BY-NC 4.0) license, which permits others to distribute, remix, adapt, build upon this work non-commercially, and license their derivative works on different terms, provided the original work is properly cited, appropriate credit is given, any changes made indicated, and the use is non-commercial. See: http://creativecommons.org/licenses/by-nc/4.0/.

ORCID iDs

Steven Law http://orcid.org/0000-0002-9335-5526

David F Hutt http://orcid.org/0000-0002-3466-306X

\section{REFERENCES}

1 Mohamed-Salem L, Santos-Mateo JJ, Sanchez-Serna J, et al. Prevalence of wild type ATTR assessed as myocardial uptake in bone scan in the elderly population. Int $J$ Cardiol 2018;270:192-6.

2 Maceira AM, Joshi J, Prasad SK, et al. Cardiovascular magnetic resonance in cardiac amyloidosis. Circulation 2005;111:186-93.

3 Fontana M, Banypersad SM, Treibel TA, et al. Native T1 mapping in transthyretin amyloidosis. JACC Cardiovasc Imaging 2014;7:157-65.

4 Rapezzi C, Quarta CC, Guidalotti PL, et al. Role of (99m)Tc-DPD scintigraphy in diagnosis and prognosis of hereditary transthyretin-related cardiac amyloidosis. JACC Cardiovasc Imaging 2011:4:659-70

5 Gillmore JD, Maurer MS, Falk RH, et al. Nonbiopsy diagnosis of cardiac transthyretin amyloidosis. Circulation 2016;133:2404-12.

6 Maurer MS, Bokhari S, Damy T, et al. Expert consensus recommendations for the suspicion and diagnosis of transthyretin cardiac amyloidosis. Circulation 2019;12:e006075.

7 Lane T, Fontana M, Martinez-Naharro A, et al. Natural history, quality of life, and outcome in cardiac transthyretin amyloidosis. Circulation 2019;140:16-26.

8 Lousada I, Maurer MS, Warner MT, et al. Amyloidosis research Consortium cardiac amyloidosis survey: results from patients with al and ATTR amyloidosis and their caregivers. J Card Fail 2019;25:S69.
9 Gillmore JD, Damy T, Fontana M, et al. A new staging system for cardiac transthyretin amyloidosis. Eur Heart J 2018:39:2799-806.

10 Cappelli F, Martone R, Gabriele M, et al. Biomarkers and prediction of prognosis in transthyretin-related cardiac amyloidosis: direct comparison of two staging systems. Can J Cardiol 2020;36:424-31

11 Rezk T, Gilbertson JA, Mangione PP, et al. The complementary role of histology and proteomics for diagnosis and typing of systemic amyloidosis. J Pathol Clin Res 2019;5:145-53.

12 Hutt DF, Fontana M, Burniston M, et al. Prognostic utility of the Perugini grading of $99 \mathrm{mTC}-\mathrm{DPD}$ scintigraphy in transthyretin (ATTR) amyloidosis and its relationship with skeletal muscle and soft tissue amyloid. Eur Heart J Cardiovasc Imaging 2017;18:1344-50.

13 Rubin J, Steidley DE, Carlsson M, et al. Myocardial contraction fraction by MMode echocardiography is superior to ejection fraction in predicting mortality in transthyretin amyloidosis. J Card Fail 2018;24:504-11.

14 Chacko L, Martone R, Bandera F, et al. Echocardiographic phenotype and prognosis in transthyretin cardiac amyloidosis. Eur Heart J 2020;41:1439-47.

15 Fontana M, Pica S, Reant P, et al. Prognostic value of late gadolinium enhancement cardiovascular magnetic resonance in cardiac amyloidosis. Circulation 2015;132:1570-9.

16 Martinez-Naharro A, Kotecha T, Norrington K, et al. Native T1 and Extracellular Volume in Transthyretin Amyloidosis. JACC Cardiovasc Imaging 2019;12:810-9.

17 Palladini G, Dispenzieri A, Gertz MA, et al. New criteria for response to treatment in immunoglobulin light chain amyloidosis based on free light chain measurement and cardiac biomarkers: impact on survival outcomes. JCO 2012;30:4541-9.

18 Kumar S, Dispenzieri A, Lacy MQ, et al. Revised prognostic staging system for light chain amyloidosis incorporating cardiac biomarkers and serum free light chain measurements. JCO 2012:30:989-95.

19 Berk JL, Suhr OB, Obici L, et al. Repurposing diflunisal for familial amyloid polyneuropathy: a randomized clinical trial. JAMA 2013;310:2658-67.

20 Maurer MS, Schwartz JH, Gundapaneni B, et al. Tafamidis treatment for patients with transthyretin amyloid cardiomyopathy. N Engl J Med Overseas Ed 2018;379:1007-16.

21 Adams D, Gonzalez-Duarte A, O'Riordan WD, et al. Patisiran, an RNAi therapeutic, for hereditary transthyretin amyloidosis. N Engl J Med Overseas Ed 2018;379:11-21.

22 Solomon SD, Adams D, Kristen A, et al. Effects of Patisiran, an RNA interference therapeutic, on cardiac parameters in patients with hereditary Transthyretin-Mediated amyloidosis. Circulation 2019;139:431-43.

23 Benson MD, Waddington-Cruz M, Berk JL, et al. Inotersen treatment for patients with hereditary transthyretin amyloidosis. N Engl J Med Overseas Ed 2018:379:22-31.

24 Dasgupta NR, Benson MD. Treatment of ATTR cardiomyopathy with a TTR specific antisense oligonucleotide, inotersen. Amyloid 2019;26:20-1.

25 Gillmore JD, Garcia-Pavia P, Grogan M. Abstract 14214: ATTRibute-CM: a randomized, double-blind, placebo-controlled, multi-center, global phase 3 study of AG10 in patients with transthyretin amyloid cardiomyopathy (ATTR-CM) 2019;140:A14214 